\title{
The BRG1 chromatin remodeling enzyme links cancer cell metabolism and proliferation
}

\author{
Qiong Wu ${ }^{1}$, Pasil Madany ${ }^{1}$, Jason R. Dobson ${ }^{1}$, Jake M. Schnable ${ }^{1}$ Soni Sharma ${ }^{2}$, \\ Tara C. Smith ${ }^{1}$ Andre J. van Wijnen ${ }^{3}$, Janet L. Stein ${ }^{4}$, Jane B. Lian ${ }^{4}$, Gary S. Stein ${ }^{4}$, \\ Rohini Muthuswami ${ }^{2}$, Anthony N. Imbalzano ${ }^{1}$, Jeffrey A. Nickerson ${ }^{1}$ \\ ${ }^{1}$ Department of Cell and Developmental Biology, University of Massachusetts Medical School, Worcester, MA, USA \\ ${ }^{2}$ School of Life Sciences, Jawaharlal Nehru University, New Delhi, Delhi, India \\ ${ }^{3}$ Department of Biochemistry and Molecular Biology, Mayo Clinic, Rochester, MN, USA \\ ${ }^{4}$ Department of Biochemistry and Vermont Cancer Center for Basic and Translational Research, University of Vermont \\ College of Medicine, Burlington, WA, USA
}

Correspondence to: Anthony N. Imbalzano, email: anthony.imbalzano@umassmed.edu Jeffrey A. Nickerson, email: jeffrey.nickerson@umassmed.edu

Keywords: breast cancer, metabolism, lipogenesis, gene regulation, BRG1

Received: January 07, 2016

Accepted: May 01, 2016

Published: May 20, 2016

\section{ABSTRACT}

Cancer cells reprogram cellular metabolism to meet the demands of growth. Identification of the regulatory machinery that regulates cancer-specific metabolic changes may open new avenues for anti-cancer therapeutics. The epigenetic regulator BRG1 is a catalytic ATPase for some mammalian SWI/SNF chromatin remodeling enzymes. BRG1 is a well-characterized tumor suppressor in some human cancers, but is frequently overexpressed without mutation in other cancers, including breast cancer. Here we demonstrate that BRG1 upregulates de novo lipogenesis and that this is crucial for cancer cell proliferation. Knockdown of BRG1 attenuates lipid synthesis by impairing the transcription of enzymes catalyzing fatty acid and lipid synthesis. Remarkably, exogenous addition of palmitate, the key intermediate in fatty acid synthesis, rescued the cancer cell proliferation defect caused by BRG1 knockdown. Our work suggests that targeting BRG1 to reduce lipid metabolism and, thereby, to reduce proliferation, has promise for epigenetic therapy in triple negative breast cancer.

\section{INTRODUCTION}

Upregulation of lipogenic genes and overall lipogenesis are hallmarks of cancer [1]. Depending on the tumor type, tumor cells synthesize up to $95 \%$ of saturated and mono-unsaturated fatty acids (FA) de novo despite sufficient exogenous supply [2]. Lipogenic enzymes such as fatty acid synthase (FASN), acetyl-CoA carboxylase (ACC), and ATP citrate lyase (ACLY) that are involved in fatty acid biosynthesis and sterol regulatory element binding protein 1 (SREBP1), the master regulator of lipogenic gene expression, are overexpressed in a number of cancers including breast, prostate, ovarian, lung, and colon [3-6]. Several lines of evidence suggest that activation of the de novo fatty acid synthesis pathway is required for carcinogenesis $[1,7,8]$. For example, elevated levels of FASN, the major enzyme responsible for fatty acid biosynthesis, are correlated with poor prognosis in breast cancer patients [1,7]. Increases in both FASN expression and activity are observed early in oncogenesis and correlate with cancer progression, with FASN-overexpressing tumors exhibiting more aggressive phenotypes [1]. Chemical or RNAi-mediated inhibition of key enzymes involved in fatty acid synthesis, including FASN, ACC and ACLY, reduces cell proliferation, induces apoptosis of cancer cells and retards the growth of human tumors in mouse xenograft models [1,9-13].

Whereas various tumor types display increased endogenous fatty acid biosynthesis irrespective of extracellular lipid availability, most normal cells, even those with comparatively high proliferation rates, preferentially use dietary/exogenous lipids for synthesis of new structural lipids $[1,12]$. We sought to investigate how lipogenic pathways are re-wired in cancer. Mammalian 
SWI/SNF complexes are evolutionarily conserved, multisubunit enzymes that mobilize nucleosomes and remodel chromatin using the energy of ATP hydrolysis [14-16]. These enzymes are important in DNA replication and repair, cell growth control, maintenance of pluripotency, and promotion of cell lineage differentiation. Increasing evidence supports an important role for human SWI/SNF enzyme subunits in cancer development $[17,18]$. Meta-analyses of cancer genome-sequencing data estimates that nearly $20 \%$ of human cancers harbor mutations in one or more SWI/SNF genes [17-20]. We and others reported that knockdown of BRG1 reduces cell proliferation in both breast epithelial and cancer cells in vitro [21-23] and attenuates tumor growth in a xenograft model $[21,22]$. However, the underlying mechanisms remained unknown. Here we report that BRG1 directly regulates triple negative breast cancer cell proliferation via regulation of lipogenic pathways. Knockdown of BRG1 decreased de novo lipid synthesis in breast cancer cells, but not in breast epithelial cells, with concomitant reduction in cell proliferation. BRG1 knockdown significantly reduced lipogenic gene expression. Chromatin immunoprecipitation analysis revealed that BRG1 was bound to sequences at lipogenic genes. Re-introducing BRG1 largely restored FASN and ACC expression, de novo lipid synthesis and cell proliferation. Supplementing the cell media with exogenous palmitate completely restored cell proliferation in BRG1 knockdown cells, thereby demonstrating a causal link between lipid synthesis and cancer cell proliferation and identifying a novel mechanism by which lipogenic signaling is crucial for cancer cell growth.

\section{RESULTS}

\section{Reduction of BRG1 in cancer cells attenuated de novo lipid synthesis}

One of the most conserved features of all cancers is the reprogramming of cellular metabolism in favor of biosynthetic processes that support high proliferation rates and survival in the tumor microenvironment [24]. To support unlimited growth, cancer cells exhibit higher rates of glucose metabolism, protein synthesis and de novo lipid synthesis $[25,26]$. We surveyed these pathways by metabolic labeling in MDA-MB-231 triple negative breast cancer cells in the presence of a scrambled sequence shRNA or shRNA targeting BRG1 [21, 22, 27]. Glucose uptake and protein synthesis were not affected in MDAMB-231 BRG1 knockdown cells (Figure 1A-1C). Interestingly, de novo lipid synthesis was reduced by $40 \%$ in the MDA-MB-231 BRG1 knockdown cells (Figure 1D) but not in MCF-10A breast epithelial cells expressing the same shRNA against BRG1 (Figure 1E). Western blot analysis confirmed the knockdown of BRG1 in both cell lines (Figure 1F). This observation was reproduced in other triple negative breast cancer lines (MDA-MB-468 and HDQ-P1) that were treated with a previously validated pool of siRNAs targeting BRG1 [22, 27] (Figure 1G-1H). ADAADi (Active DNA-dependent ATPase A Domain inhibitor), a minor product generated by the bacterial APH (3')-III enzyme that encodes for aminoglycoside resistance, inhibits the ATPase activity of the SWI2/SNF2 family of ATPases [28, 29] and increases the chemosensitivity of triple negative breast cancer cells to clinically relevant therapeutic drugs [30]. Pharmacological inhibition of the BRG1 ATPase domain by ADAADi in MDA-MB231 cells also decreased de novo lipid synthesis (Figure 1I). Collectively, the data show a role for BRG1 in promoting de novo lipid synthesis in triple negative breast cancer cells.

\section{BRG1 upregulates lipogenic gene expression in triple negative breast cancer cells}

The reduction in de novo lipid synthesis in BRG1 knockdown breast cancer cells was directly caused by reduction of lipogenic gene expression. De novo fattyacid synthesis involves two key enzymes, ACC and FASN. ACC carboxylates acetyl-CoA to form malonyl-CoA, and the malonyl-CoA product is subsequently converted by FASN to palmitate, a precursor for longer-chain fatty acids [31]. As shown in Figure 2A, mRNA levels of ACC and FASN were down-regulated after BRG1 knockdown in breast cancer cells but not in non-tumorigenic MCF-10A mammary epithelial cells. The protein levels of these enzymes were also reduced by BRG1 knockdown in breast cancer cells, but not in non-tumorigenic MCF-10A mammary epithelial cells (Figure 2B).

The expression of other genes involved in the synthesis or metabolism of lipids was also regulated by BRG1 (Figure 2C). Elevated glucose catabolism produces an excess of the glycolytic end-product, pyruvate. Most excess pyruvate is converted to lactate, but some is converted to acetyl-CoA by ATP citrate lyase (ACLY) and can be used in de novo fatty-acid synthesis. Therefore, ACLY is the link between the metabolism of carbohydrates and the production of fatty acids and represents an important step in fatty acid biosynthesis [32]. Longchain acyl CoA synthetase (ACSL) catalyzes the first step in fatty acid activation for intracellular metabolism by converting long-chain fatty acids into acyl-CoA thioesters $[33,34]$. ACSL1 is the best-studied and the major ACSL isoform, is highly expressed in major energy-metabolizing tissues, and plays a key role in lipid biosynthesis and fatty acid degradation [35]. Lipin-1 (LPIN1) is a magnesiumdependent phosphatidic acid phosphohydrolase that catalyzes the penultimate step in triglyceride synthesis including the dephosphorylation of phosphatidic acid to yield diacylglycerol [36]. The expression of all these genes was decreased by BRG1 knockdown (Figure 2C).

We next showed that BRG1 directly bound to these genes having BRG1-dependent expression. We predicted BRG1 binding sites based on BRG1 ChIP-seq 

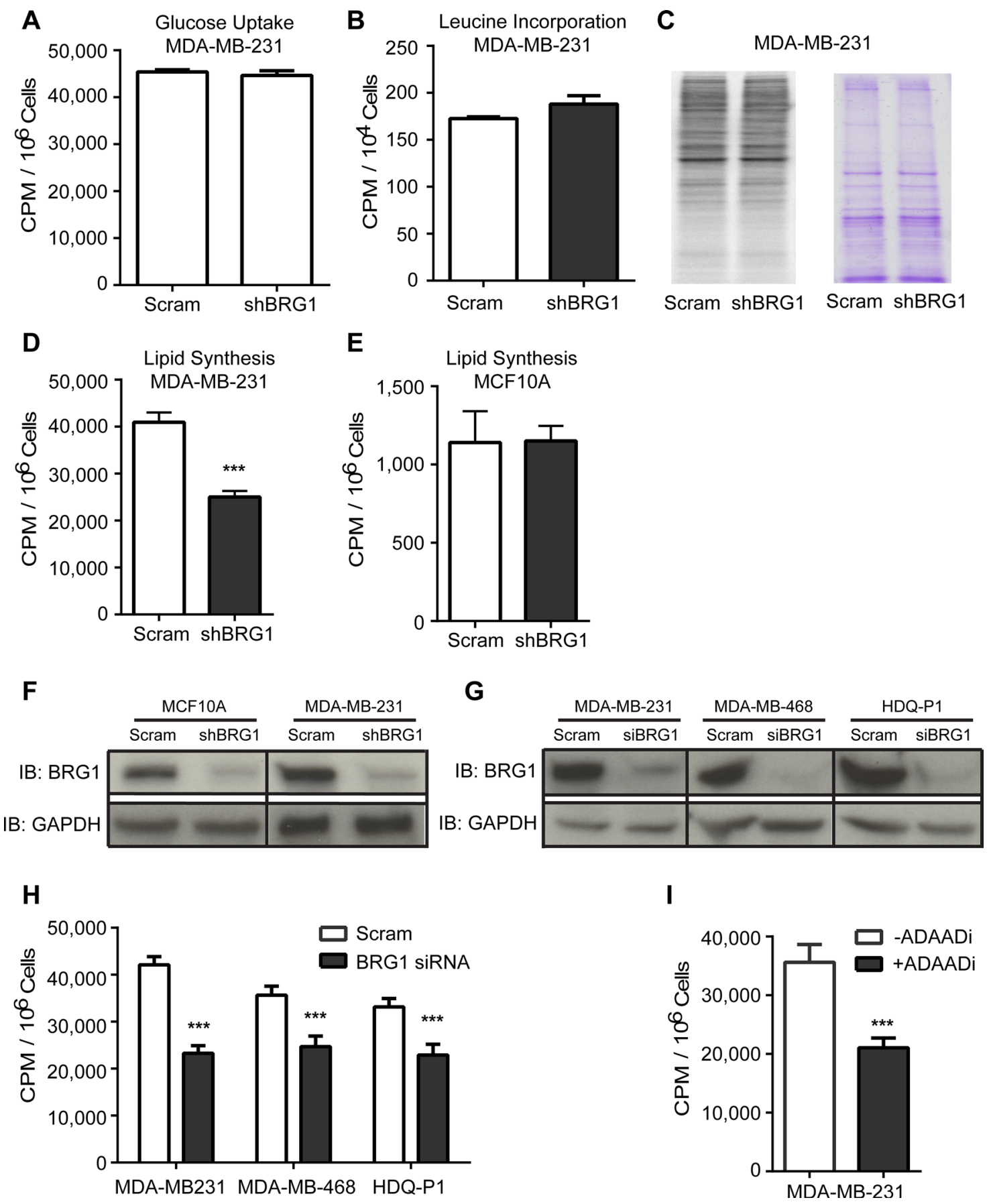

Figure 1: BRG1 knockdown reduced de novo lipid synthesis in triple negative breast cancer cells but not in MCF-10A mammary epithelial cells. (A) MDA-MB-231 cells expressing either a scrambled sequence shRNA or an shRNA targeting BRG1 were incubated with ${ }^{14} \mathrm{C}$-glucose and incorporation of radioactivity into cells was measured to determine glucose uptake. There was no significant change. (B) BRG1 knockdown cells also had no change in the rate of protein synthesis compared to control cells as measured by ${ }^{3} \mathrm{H}$-leucine incorporation. (C) Phosphorimage of the blot of ${ }^{35} \mathrm{~S}$-labeled protein extracted from control and BRG1 knockdown cells (left) and Coomassie brilliant blue staining of the blot (right). (D) BRG1 knockdown cells had a decrease in de novo lipid synthesis as measured by ${ }^{14} \mathrm{C}$-acetate incorporation in total extracted lipids. (E) MCF-10A cells expressing a scrambled sequence shRNA or shRNA targeting BRG1 were incubated with ${ }^{14} \mathrm{C}$-acetate and incorporation of radioactivity into extracted total lipids was measured. BRG1 knockdown did not cause a significant decrease in lipid synthesis. (F) Western blot analysis verified the shRNA-mediated knockdown of BRG1 in MDAMB-231 and MCF-10A cells. (G) Western blot analysis verified the siRNA-mediated knockdown of BRG1 in MDA-MB-468 and HDQ-P1 cells. (H) Three triple negative breast cancer cell lines were treated with scrambled sequence siRNA or a cocktail of siRNAs targeting BRG1 and ${ }^{14} \mathrm{C}$-acetate incorporation into extracted total lipids was measured. (I) A small molecule inhibitor (ADAADi) of BRG1 inhibited de novo lipid synthesis in MDA-MB-231 cells. Each data point represents the mean of 3 independent experiments performed in triplicate. Error bars are standard deviations. $* * * P<0.001$. 
data from K562 and HeLa cells and global H3K27Ac and DNase I hypersensitivity analyses [37] and used these for PCR primer design within the region $1.5 \mathrm{~Kb}$ upstream of the TSS of the ACC, FASN, ACLY and ACSL1 genes (Supplementary Figure 1). There were no active transcriptional marks in sequences upstream of the LPIN1 TSS, however, there were multiple active marks in the first intronic region of this gene, allowing the design of PCR primers in this region (Supplementary Figure 1). Chromatin immunoprecipitation (ChIP) analysis detected BRG1 binding at these lipogenic genes in control cells that was markedly decreased in BRG1 knockdown cells (Figure 2D). Additional ChIP controls are shown in Supplementary Figure 2. These data suggest that BRG1 directly and transcriptionally regulates lipid biosynthesis and metabolism pathways in these breast cancer cells.

\section{Reduction in de novo lipid synthesis impaired cancer cell proliferation}

To further confirm that BRG1 is the direct cause for the reduction in lipid biosynthesis, a complementary experiment was performed to restore BRG1 expression in the knockdown cells. Re-expression or over-expression of wildtype or catalytically inactive BRG1 is negligible in some cell types if Brahma (BRM), the closely related ATPase that can act as the catalytic subunit in mammalian SWI/SNF complexes in a manner that is mutually exclusive with BRG1, is expressed [38-40]. In addition, we have previously shown that knockdown of either BRG1 or $\mathrm{BRM}$ in triple negative breast cancer cell lines results in increased expression of the remaining ATPase [22] and that both BRG1 and BRM contribute to triple negative cell proliferation [30]. Therefore we re-established BRG1 expression in MDA-MB-231 cells after expressing shRNA against both BRG1 and BRM [22] by transient transfection with plasmid vectors. Re-expression of BRG1 in this double knockdown background resulted in increasing levels of FASN and in a dose-dependent manner (Figure 3A), as well as a concomitant increase in de novo lipid synthesis (Figure 3B). As we previously reported [22], re-expression of BRG1 in double knockdown cells caused only a partial rescue in cell proliferation (Figure 3C). This is expected since we have shown that BRM also contributes to cell proliferation [30]. These data show that BRG1 regulates both de novo lipid synthesis and cell proliferation.

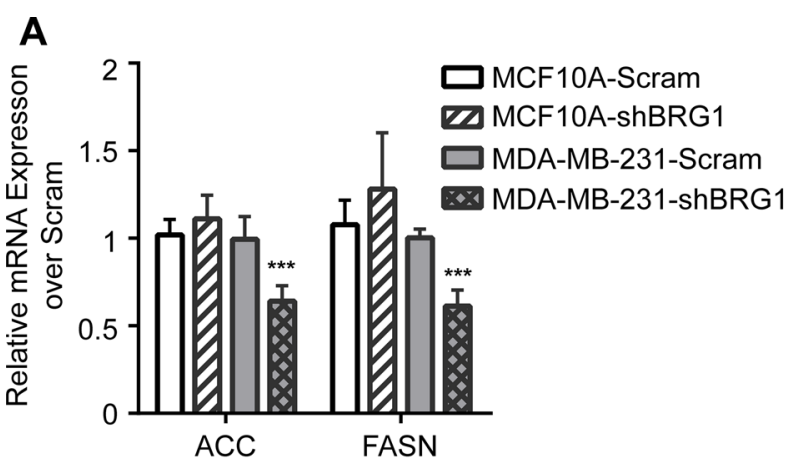

\section{B}
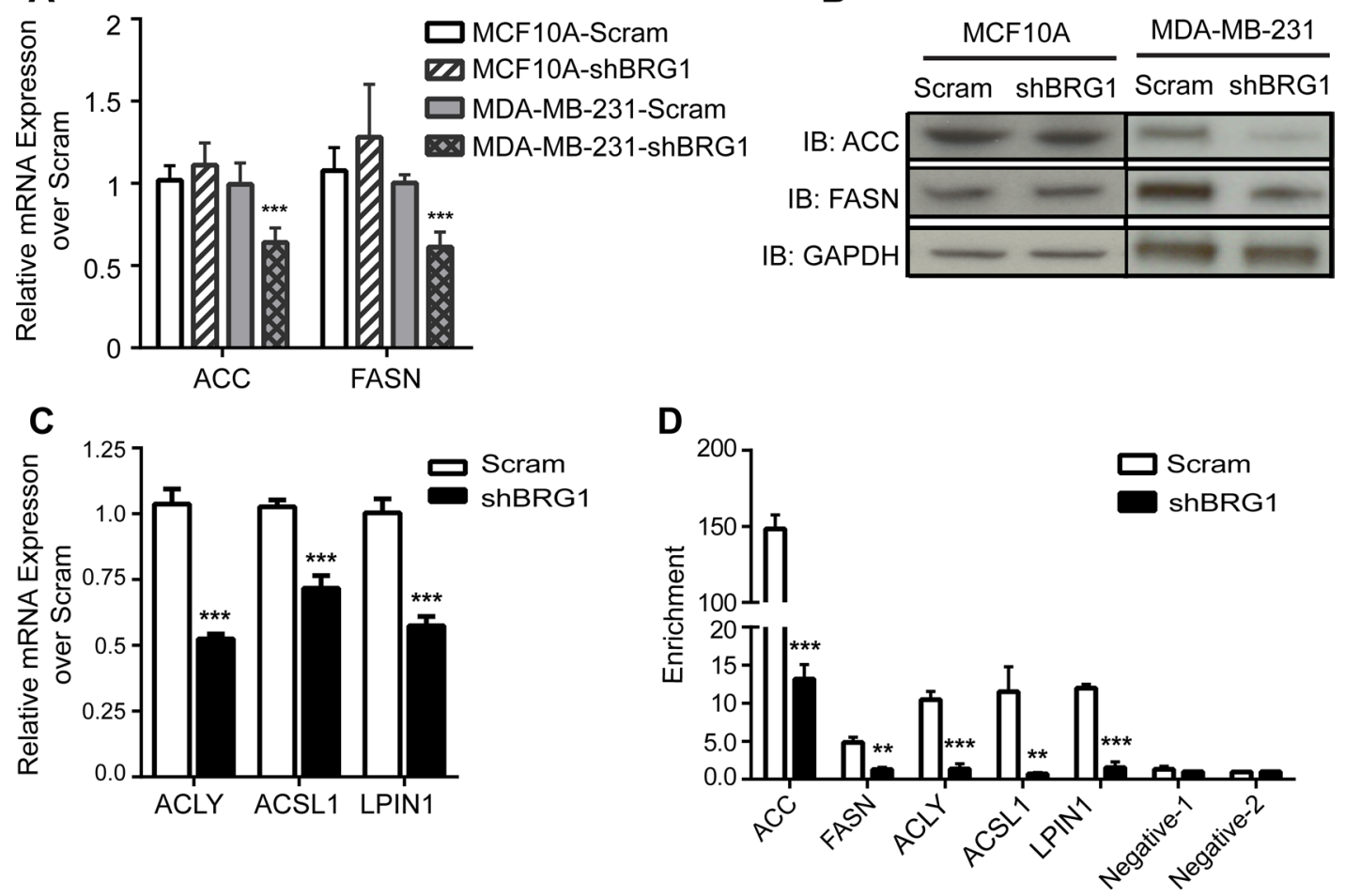

Figure 2: BRG1 was required for the expression of genes involved in fatty acid and lipid synthesis. (A) mRNA levels of ACC and FASN in MDA-MB-231 and MCF-10A cells expressing either a scrambled sequence shRNA or an shRNA targeting BRG1 were measured by qPCR. (B) Western blot analysis measuring protein levels of ACC and FASN in MDA-MB-231 and MCF-10A cells expressing a scrambled sequence shRNA or shRNA targeting BRG1. (C) mRNA levels of ACLY, ACSL1 and LPIN1 in MDA-MB-231 cells expressing either a scrambled sequence shRNA or an shRNA targeting BRG1 were determined by qPCR. (D) ChIP experiments with MDA-MB-231 cells expressing either a scrambled sequence shRNA or an shRNA targeting BRG1 demonstrated that BRG1 binds to sequences upstream of each gene. Negative control sequences 1 and 2 are within the coding sequences of the ACC and FASN genes, respectively. Each dataset represents the mean of 3 independent experiments performed in triplicate. Error bars are standard deviations. $* * P<0.01, * * * P<0.001$. 
We reasoned that, as an upstream regulator of key lipogenic enzymes, depletion of BRG1 should also increase the sensitivity of cells to fatty acid synthesis inhibitors. 5-tetradecyloxy-2-furoic acid (TOFA) is an inhibitor of ACC [41]. When TOFA was added to cells, the decrease in viable cell number was larger after BRG1 knockdown than in control cells (Figure 4A). The FASN inhibitor c75 [42] decreased cell viability in control cells, underscoring the need for de novo lipid biogenesis in highly proliferative tumor cells. As shown in Figure 4B, c75 potency was significantly enhanced in BRG1 knockdown cells compared to control cells. ACC and FASN have been reported to be essential to cancer cell survival, and knocking down either ACC or FASN dramatically decreases cancer cell proliferation [9, 43, 44]. After reducing BRG1 levels, breast cancer cells showed increased sensitivity to both inhibitors.

Since the end product of FASN and common intermediate in all de novo fatty acid synthesis is palmitate, we asked if the decrease in palmitate production was responsible for the impaired cell proliferation in

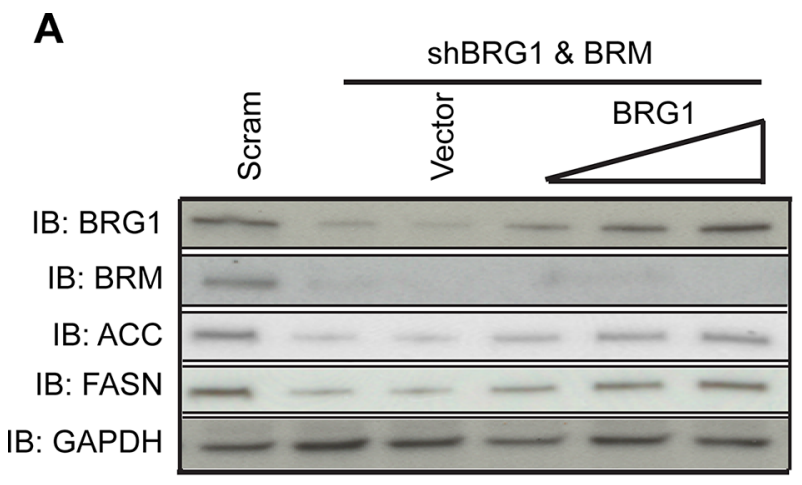

C

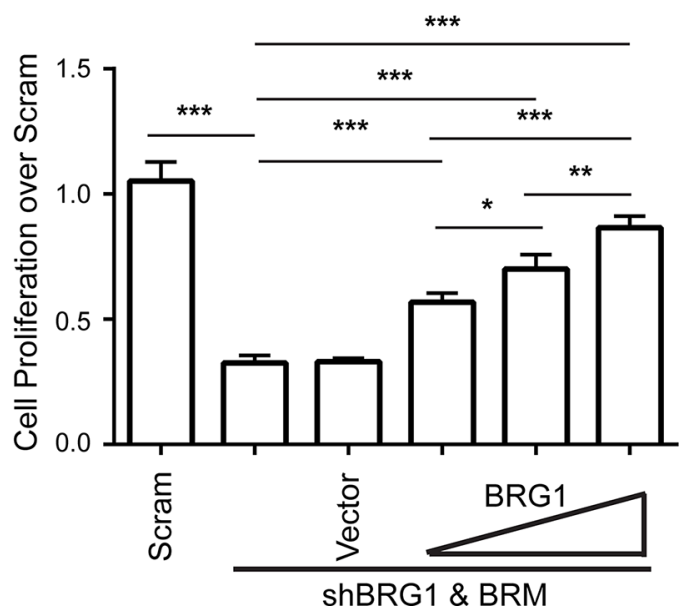

cancer cells with BRG1 knockdown [22]. Following BRG1 knockdown, the cell culture medium was replaced with medium containing increasing doses of nonfat BSA-conjugated palmitic acid, and cell proliferation was measured. As shown in Figure 4C, addition of exogenous palmitic acid in the culture medium completely rescued the cell proliferation defect in BRG1 knockdown cells but had no effect on control cells. This result shows that BRG1dependent contributions to de novo lipid synthesis in turn regulate the rate of the breast cancer cell proliferation.

Addition of palmitate did not affect ACC or FASN expression in either control of BRG1 knockdown cells (Figure 4D). Thus BRG1 function is upstream of ACC and FASN expression, which is upstream of palmitate production and de novo lipid synthesis, which are upstream of cell proliferation. The data do not exclude possible feedback signals whereby the altered proliferation rate also affects de novo lipid synthesis. Regardless, the results demonstrate that decreased cell proliferation in BRG1 knockdown cells can be attributed to the decrease in expression of the key metabolic enzymes for fatty acid synthesis.

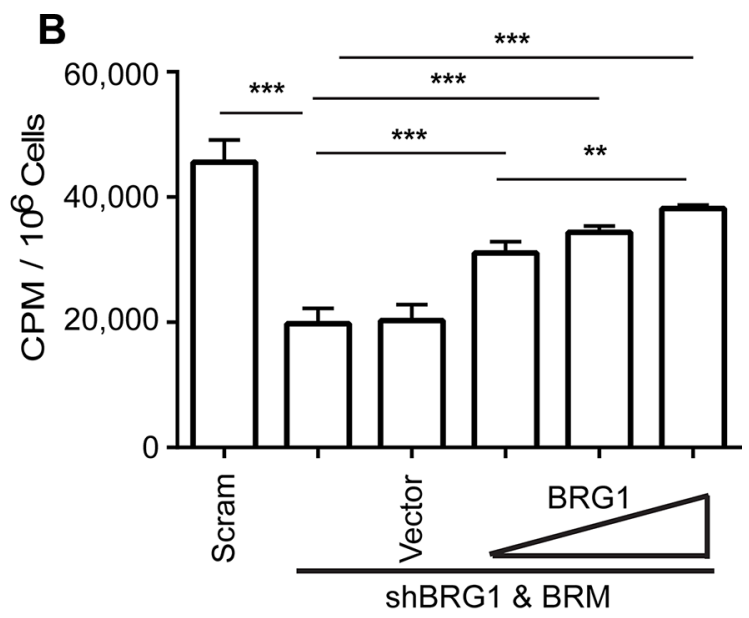

Figure 3: Restoration of BRG1 expression in cells depleted for BRG1 partially rescued the decrease in de novo lipid synthesis and cell proliferation. (A) Western blot analysis showing that re-expression of BRG1 partially restored ACC and FASN protein levels. (B) Re-expression of BRG1 partially reversed the inhibition of de novo lipid synthesis caused by loss of BRG1. (C) Re-expression of BRG1 partially reversed the inhibition of cell growth caused by loss of BRG1. Each dataset represents the mean of 3 independent experiments performed in triplicate. Error bars are standard deviations. $* P<0.05, * * P<0.01, * * * P<0.001$. 


\section{DISCUSSION}

One of the hallmarks of cancer is elevated de novo fatty acid synthesis [45]. Clinical and basic scientific investigation has shown that human cancers synthesize fatty acids via the de novo fatty acid synthesis pathway even when exogenous fatty acids are abundant, seemingly independent of the regulatory signals that control fattyacid synthesis in normal cells. The role of FASN in normal human biology includes energy storage from excess carbohydrates to fat in liver and adipose tissue and specialized functions that facilitate lactation in the breast and reproduction in endometrium and decidua [46-48]. FASN expression during these processes is strictly regulated by nutrition and hormonal levels [49]. In contrast, FASN is highly expressed in many cancers and precancerous lesions. In this context, the expression of FASN is independent of nutrition, and in many cases, it appears independent of hormonal regulation [45]. Despite its promise as a target for anti-cancer therapeutics $[1,12$, $50,51]$, the mechanism by which FASN is dysregulated in cancer is unknown. Similarly, two enzymes catalyzing rate limiting steps upstream of FASN in de novo fatty acid synthesis, ACC and ACLY, are often dysregulated in cancer and have been proposed as breast cancer therapeutic targets [52-54].

BRG1 is a known epigenetic regulator of chromatin structure and gene expression that may also play an architectural role in gene organization [55]. We show here that loss of BRG1 attenuated FASN, ACC, and ACLY expression and impaired de novo lipid synthesis in breast cancer cells with a concomitant decrease in cell proliferation. Importantly, this phenomenon was only seen in cancer cells and not in non-tumorigenic mammary epithelial cells. Re-introducing BRG1 to BRG1- and BRM-depleted cancer cells increased FASN and ACC expression, increased de novo lipid synthesis, and partially restored cell proliferation. When the culture medium was
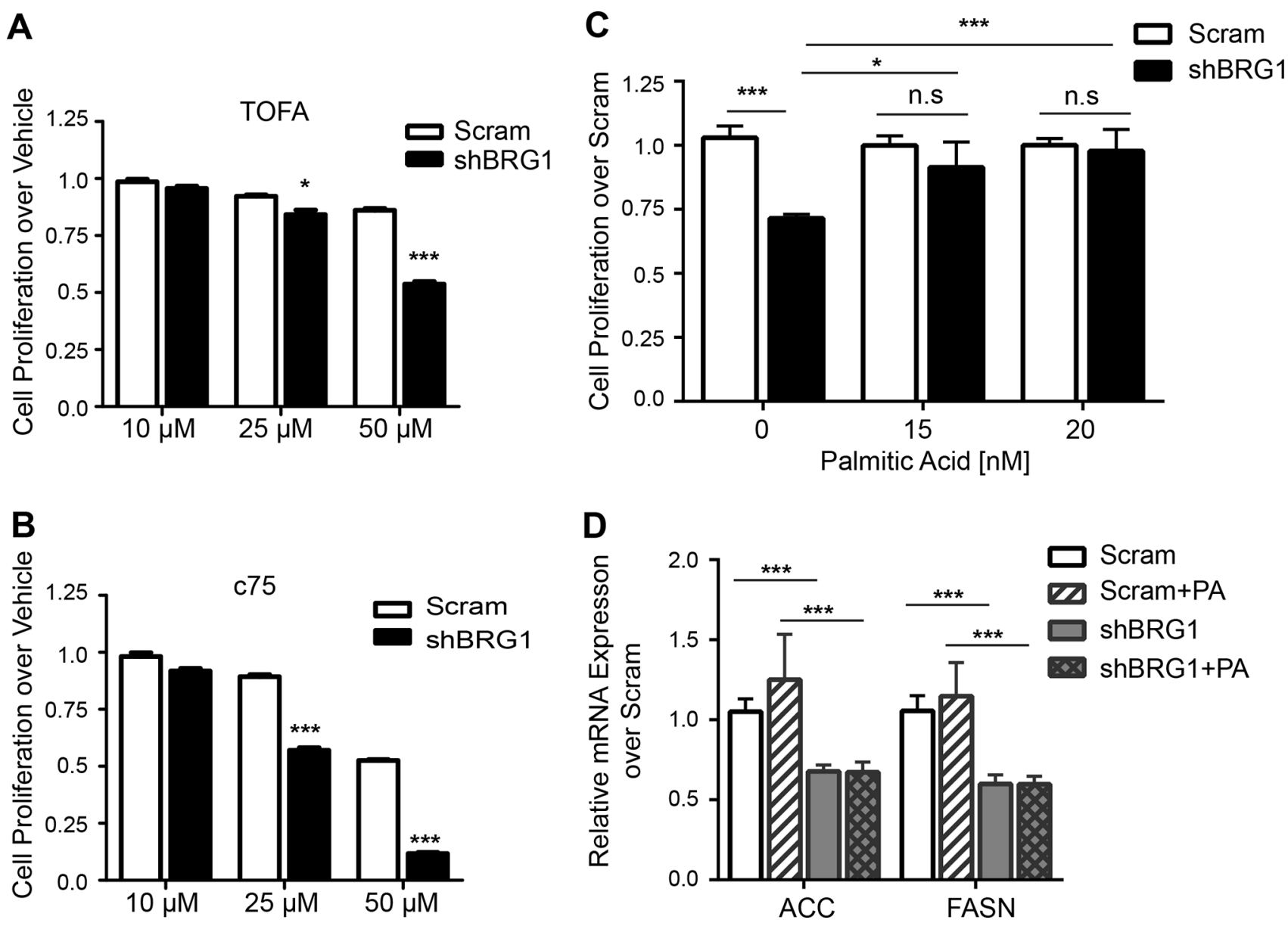

Figure 4: Fatty acid levels regulated breast cancer cell proliferation. (A-B) BRG1 knockdown rendered cells more sensitive to growth inhibition by the ACC inhibitor TOFA or the FASN inhibitor c75. (C) Addition of palmitic acid to the cell culture media completely reversed the growth inhibition caused by BRG1 knockdown. (D) Addition of palmitic acid did not affect FASN or ACC expression in control or in BRG1 knockdown cells. Each dataset represents the mean of 3 independent experiments performed in triplicate. Error bars are standard deviations. $* P<0.05, * * * P<0.001$, n.s. not significant. 
supplemented with palmitic acid, the end product of FASN and the key intermediate in the synthesis of longer chain and desaturated fatty acids, cell proliferation in BRG1 knockdown cells was completely rescued. The complete rescue in cells where only BRG1 was knocked down and BRM was present suggests that BRG1 is the SWI/ SNF ATPase predominantly responsible for regulation of these metabolic pathways. Thus our results provide the first evidence of a direct relationship between BRG1, lipogenic enzyme transcriptional control, de novo fatty acid synthesis, and cell proliferation.

We and others have demonstrated that BRG1 levels are elevated in primary breast cancer [22, 23]. Our results here show that the elevated levels of lipid synthesis found in breast cancer are dependent on BRG1 acting to upregulate the transcription of FASN, ACC, ACLY and other genes involved in fatty acid synthesis. BRG1 is therefore an epigenetic link between breast cancer cell proliferation and fatty acid synthesis, but it is likely to be only the first factor identified in a novel regulatory circuit up-regulating lipogenic enzyme expression in cancer with downstream consequences on cell proliferation. Further investigation of BRG1 and the BRG1-interacting factors of this circuit will elucidate mechanisms by which cancer cells rewire signaling pathways controlling de novo lipid biosynthesis.

\section{Strategically targeting BRG1 for cancer therapy}

BRG1 function in cancer appears to be context dependent. It is mutated in lung and other cancers [56, 57], and cancers featuring loss of the SNF5/INI1 subunit may require BRG1 [18], thereby suggesting the potential of targeting BRG1 to treat such tumors [58, 59]. In addition, BRG1 is upregulated with little evidence of mutation in primary breast and prostate tumors, in melanoma and neuroblastoma, and in pancreatic, gastric, and colorectal carcinomas [22, 23, 60-67]. Therefore, developing small molecule inhibitors that interfere with BRG1 function or that fine-tune the expression of BRG1 back to physiological levels might provide therapeutic benefits. Indeed, we have recently demonstrated that knockdown of BRG1 or use of a BRG1 inhibitor sensitizes triple negative breast cancer cells to commonly used chemotherapeutic agents, perhaps via modulation of $\mathrm{ABC}$ transporter expression [30].

The data presented here show that BRG1 is specifically required for fatty acid and lipid synthesis in triple negative breast cancer cells but not in mammary epithelial cells. This specificity increases the promise for BRG1- based therapies in triple negative breast cancer compared to treatment approaches that non-specifically target fatty acid and lipid synthesis. We conclude that targeting BRG1 and its cooperating factors are a promising and novel strategy for attacking both cancer cell proliferation and cancer metabolism.

\section{MATERIALS AND METHODS}

\section{Cell culture}

MCF-10A cells from the Karmanos Cancer Institute (Detroit, MI) were maintained in monolayer as described [68]. MDA-MB-231 cells were obtained from T. Guise [69]. MCF-10A cells inducibly expressing shRNA targeting BRG1 or a control shRNA were previously described [21]. MDA-MB-231 cells inducibly expressing shRNA targeting BRG1, BRG1 + BRM, or a control shRNA were previously described [22]. MDA-MB-468 cells were obtained from Professor Hong Zhang (UMass Medical School). HDQ-P1 cells were purchased from the Leibniz Institute DSMZGerman Collection of Microorganisms and Cell Culture (Braunschweig, Germany). MDA-MB-231, MDA-MB-468 and HDQ-P1 cells were maintained in DMEM containing 10\% FBS and Penicillin/Streptomycin. Doxycyclineinducible knockdown was performed as described [21, $22,27]$ using $500 \mathrm{ng} / \mathrm{ml}$ doxycycline for MCF-10A cells and $100 \mathrm{ng} / \mathrm{ml}$ doxycycline for MDA-MB-231 cells. siRNA-mediated knockdown of BRG1 was performed using the validated pool of siRNAs and methods described previously $[22,27]$.

The identities of the breast cancer cell lines were authenticated by Short Tandem Repeat profiling at the Genetic Resources Core Facility, Johns Hopkins School of Medicine, Institute of Genetic Medicine. MCF-10A cells were similarly authenticated at the University of Vermont Cancer Center.

\section{Reagents}

Doxycycline, palmitic acid, TOFA, c75, MTT (3-(4, 5-dimethylthiazolyl-2)-2,5-diphenyltetrazolium bromide), and anti-GAPDH antibody were purchased from Sigma (Sigma-Aldrich, St. Louis, MO). FASN (C20G5) and acetylCoA carboxylase (C83B10) antibodies were purchased from Cell Signaling (Cell Signaling Technology, Inc., Danvers, MA, USA). Anti-rabbit and anti-mouse IgG, and HRP-linked antibodies were from GE (GE Healthcare Life Science, Pittsburgh, PA, USA). BRG1 antiserum [70] was used in western blot and ChIP experiments. The BRM antibody (ab15597) used in western blots was purchased from Abcam (Cambridge, MA, USA). D- ${ }^{14} \mathrm{C}-$ Glucose, ${ }^{3} \mathrm{H}$-Leucine, ${ }^{35} \mathrm{~S}-$ Methionine and $\left[2-{ }^{14} \mathrm{C}\right]$ acetic acid were purchased from PerkinElmer (PerkinElmer Life Sciences, Waltham, MA, USA). ADAADi was prepared as described [28].

\section{Proliferation assays}

Five thousand cells were seeded in each well of 96-well plates, and treated as indicated in each figure legend the following day. MTT solution at a final concentration of $5 \mathrm{ug} / \mathrm{mL}$ was added to each well and samples were incubated for 4 hours. The media was 
subsequently removed and the plates were air-dried. One hundred microliters of DMSO were added to each well and incubated for 30 minutes at room temperature with gentle shaking. Absorbance was measured at OD540 in a Synergy H4 Hybrid microplate reader (Bio Tek, Winooski, VT).

\section{Glucose uptake assay}

Cells were pulse labeled with $1 \mu \mathrm{Ci}{ }^{14} \mathrm{C}$-glucose for 5 minutes, and then washed 3 times with PBS. Cells were trypsinized and counted. One million cells were lysed by adding $200 \mu \mathrm{L} 0.2 \mathrm{~N} \mathrm{NaOH}$ for $15 \mathrm{~min}$. The cell lysate were transferred to a scintillation vial, and radioactive signal was measured in a scintillation counter (Beckman Coulter LS6500).

\section{Protein synthesis assays}

Protein synthesis rate was determined by labeling cells with $1 \mu \mathrm{Ci}^{3} \mathrm{H}$-Leucine for $4 \mathrm{~h}$. After three washes in ice cold PBS, cells were lysed with PBS containing $2 \%$ SDS before scintillation counting. All readings were normalized by cell number determined from parallel plates. For other protein synthesis assays, cells were labeled with $10 \mu \mathrm{Ci}{ }^{35} \mathrm{~S}-\mathrm{Methionine}$ overnight, washed 3 times with ice cold PBS, and then lysed with PBS containing 1\% NP-40. Radiolabeled proteins $(2 \mu \mathrm{g})$ were run on SDS-PAGE 4-20\% gradient gels before transfer to a PVDF membrane. Radioactivity was measured by Phosphoimager and checked for equal protein loading by staining the membrane with Coomassie Brilliant Blue.

\section{De novo lipid synthesis assay}

In experiments with $\mathrm{ADAADi}$, cells were pretreated with $2 \mu \mathrm{M}$ ADAADi for $48 \mathrm{~h}$ prior to addition of radiolabeled acetate. Breast cancer cells were labeled with $1 \mu \mathrm{Ci} / \mathrm{mL}{ }^{14} \mathrm{C}$-acetate for $1 \mathrm{~h}$. MCF-10A cells were labeled with $5 \mu \mathrm{Ci} / \mathrm{mL}^{14} \mathrm{C}$ acetate for $1 \mathrm{~h}$. After removal of the labeling medium, cells were washed 3 times with PBS and then cultured in fresh medium for $24 \mathrm{~h}$. At the end of the incubation, cells were trypsinized and counted. One million cells were used for lipid extraction. Total lipids were extracted as described [71], transferred to a scintillation vial and counted in a scintillation counter (Beckman Coulter LS6500).

\section{c75, TOFA and palmitic acid treatment}

MDA-MB-231 SCRAM and shBRG1 cells were plated in 96-well plates at a density of 3,000 cells/well and treated with $0.1 \mu \mathrm{g} / \mathrm{ml}$ doxycycline or with vehicle for 48 h. Reagents were added to the cell culture medium at 0,15 , or $20 \mathrm{nM}$ for $24 \mathrm{~h}$ (palmitic acid) or at 10,25 , or $50 \mu \mathrm{M}$ for $72 \mathrm{~h}$ (c75, TOFA). To assess cell proliferation, $10 \mu \mathrm{L}$ of MTT reagent was added to each well and incubated for $4 \mathrm{~h}$ prior to an MTT assay.

\section{Western blotting}

Cells were washed, trypsinized, and counted and whole cell lysates from one million cultured cells were prepared by lysis in $200 \mu \mathrm{L}$ of $1 \times$ Laemmli sample buffer and boiled for $5 \mathrm{~min} .10 \mu \mathrm{L}$ of lysate was separated on SDS-polyacrylamide gels (4-20\% gradient) and transferred to PVDF membrane. Membranes were blocked in 5\% non-fat dry milk in PBS, incubated with primary antibodies overnight at $4^{\circ} \mathrm{C}$. Following repeated washing in $5 \%$ milk/PBS, membranes were incubated with secondary antibody conjugated to HRP for 1 hour at room temperature, washed repeatedly with $5 \%$ milk/PBS, and developed using Amersham ECL Western Blotting Detection Reagents and Amersham Hyperfilm ECL (GE LifeScience).

\section{RT-qPCR}

One million cells were used for extraction of total RNA using RNeasy Plus following manufacturer's instructions (Qiagen Inc., Valencia, CA, USA). cDNA synthesis was accomplished using a SuperScript III kit (Invitrogen, San Diego, CA, USA). Gene expression was measured by real time qPCR using following primers. ACC alpha (ACACA) forward 5'-ATGCG GTCTATCCGTAGG3', reverse 5'-GGTGTGACCATGA CAAC-3'; FASN, forward 5'-GTTTGATGCCTCCTTCTTC-3', reverse 5'-CGGAG TG AATCTGGGTTG-3'; ACLY forward 5'- CCCAAGTCCAAGATCCCTGC-3', reverse 5'TCGTCTCGG GAGCAGACATA-3'; ACSL1 forward 5'- GGAAGAGCCAACAGA CGGAA-3', reverse 5'CATTGCTCCTTTGGGGTTGC-3'; LPIN1 forward 5'- GAGGCAGAC AGCACCATACA-3', reverse 5'GGCTAACTGCCCCACGTAAT-3'.

\section{Chromatin immunoprecipitation (ChIP)}

ChIP was performed as described previously [38] with modifications. Cells were cooled to room temperature before crosslinking which was done by replacing the media with ice-cold growth medium containing $3.7 \%$ formaldehyde for $40 \mathrm{~min}$ at $4^{\circ} \mathrm{C}$. Each ChIP reaction used $5 \mu \mathrm{g}$ of BRG1 antiserum [70] or control IgG (Millipore, Billerica, MA) and 50 $\mu \mathrm{g}$ of chromatin extract. Primers used for measuring BRG1 binding at those promoters measured by qPCR are listed below: ACC alpha (ACACA), forward 5'GTCCCACCCCGTAAGGATTT $-3^{\prime}(-537 \sim-556$ bp to TSS), reverse 5'- GGCGCTAGCTCCAAACTAAC -3' (-708 -727 bp to TSS);

FASN, forward 5'- CTCCCGAGTGATTCCTCGAA $-3^{\prime}(-1086 \sim-1105$ bp to TSS), reverse 5'- CTCAAAGT AGGACACGCAGC -3' (-1233 -1252 bp to TSS);

ACLY, forward 5'- GTAAGCAAGTGGGGCTAGG AG $-3^{\prime}(-570 \sim-590$ bp to TSS), reverse 5'CTTCGCT GGAATCTCGCATTG -3' (-665 -684 bp to TSS); 
ACSL1, forward 5'- CCAGACTGCCTCGGA TTTCATA $-3^{\prime}(-130 \sim-151$ bp to TSS), reverse 5'GGCGGTCCAATGTACCCTT

$-3^{\prime}(-172 \sim-191$ bp to TSS);

LIPIN1, forward 5'-TGCAGCCCATTTCCTGG ATT-3' $(+66,769$ bp $\sim+66,788$ bp to TSS $)$, reverse 5'-GAGGAAGGAGGGGCTGAGTA-3' (+66,842 bp (+66,861 bp to TSS).

Genomic region (chr17:35,716,490-35,716,996) near the ACACA promoter was used as a negative control for BRG1 binding, forward 5'-ATACATGC TGGATCCTGGCG-3', reverse 5'-GGACGGGAAGCA TTCTCCAA-3'. Genomic region (chr17:80,055,818$80,056,106)$ near the FASN promoter served as an additional negative control for BRG1 binding, forward 5'-CTCCGAAGGGGCACGAAC-3', reverse 5'-TCCTC ATCCTCCGCTCTCG-3'. Meanwhile, ABCC3 gene expression was not affected by depletion of BRG1 in our previous work [30]. Therefore BRG1 binding at this locus was used to serve additional negative control, with primer sequences as following: forward 5'ATTCAGGAGGGAGCTTTGCC-3' (+4,555 bp +4,754 bp to TSS), reverse 5'- CCATTTCCCTGTCTGGGGAC$3^{\prime}(+4,589$ bp $\sim+4,608$ bp to TSS). RNase polymerase II binding at this locus was used as positive control in the ChIP experiment.

\section{Statistical analyses}

Quantified data represent the mean of three independent experiments performed in triplicate with standard deviation (S.D). Statistical relevance was evaluated using GraphPad Instat two-tail $P$ value student test (Graphpad Software, Inc., La Jolla, CA).

\section{ACKNOWLEDGMENTS}

We thank members of the Imbalzano lab group for comments.

\section{CONFLICTS OF INTEREST}

The authors declare that they have no conflicts of interest.

\section{GRANT SUPPORT}

This work was supported by NIH grants P01 CA82834, R01 EB014869, and R21 CA185926.

\section{REFERENCES}

1. Menendez JA, Lupu R. Fatty acid synthase and the lipogenic phenotype in cancer pathogenesis. Nat Rev Cancer. 2007; 7:763-777.
2. Vazquez-Martin A, Colomer R, Brunet J, Lupu R, Menendez JA. Overexpression of fatty acid synthase gene activates HER1/HER2 tyrosine kinase receptors in human breast epithelial cells. Cell Prolif. 2008; 41:59-85.

3. Martel PM, Bingham CM, McGraw CJ, Baker CL, Morganelli PM, Meng ML, Armstrong JM, Moncur JT, Kinlaw WB. S14 protein in breast cancer cells: direct evidence of regulation by SREBP-1c, superinduction with progestin, and effects on cell growth. Exp Cell Res. 2006; 312:278-288.

4. Migita T, Narita T, Nomura K, Miyagi E, Inazuka F, Matsuura M, Ushijima M, Mashima T, Seimiya H, Satoh Y, Okumura S, Nakagawa K, Ishikawa Y. ATP citrate lyase: activation and therapeutic implications in non-small cell lung cancer. Cancer Res. 2008; 68:8547-8554.

5. Mukherjee A, Wu J, Barbour S, Fang X. Lysophosphatidic acid activates lipogenic pathways and de novo lipid synthesis in ovarian cancer cells. J Biol Chem. 2012; 287:24990-25000.

6. Verhoeven G. Androgens and increased lipogenesis in prostate cancer. Cell biologic and clinical perspectives. [Article in Dutch]. Verh K Acad Geneeskd Belg. 2002; 64:189-195; discussion 195-186.

7. Kuhajda FP, Jenner K, Wood FD, Hennigar RA, Jacobs LB, Dick JD, Pasternack GR. Fatty acid synthesis: a potential selective target for antineoplastic therapy. Proc Natl Acad Sci U S A. 1994; 91:6379-6383.

8. Zhou W, Han WF, Landree LE, Thupari JN, Pinn ML, Bililign T, Kim EK, Vadlamudi A, Medghalchi SM, El Meskini R, Ronnett GV, Townsend CA, Kuhajda FP. Fatty acid synthase inhibition activates AMP-activated protein kinase in SKOV3 human ovarian cancer cells. Cancer Res. 2007; 67:2964-2971.

9. Chajes V, Cambot M, Moreau K, Lenoir GM, Joulin V. Acetyl-CoA carboxylase alpha is essential to breast cancer cell survival. Cancer Res. 2006; 66:5287-5294.

10. Hatzivassiliou G, Zhao F, Bauer DE, Andreadis C, Shaw AN, Dhanak D, Hingorani SR, Tuveson DA, Thompson CB. ATP citrate lyase inhibition can suppress tumor cell growth. Cancer Cell. 2005; 8:311-321.

11. Kridel SJ, Axelrod F, Rozenkrantz N, Smith JW. Orlistat is a novel inhibitor of fatty acid synthase with antitumor activity. Cancer Res. 2004; 64:2070-2075.

12. Mashima T, Seimiya H, Tsuruo T. De novo fatty-acid synthesis and related pathways as molecular targets for cancer therapy. Br J Cancer. 2009; 100:1369-1372.

13. Zaidi N, Swinnen JV, Smans K. ATP-citrate lyase: a key player in cancer metabolism. Cancer Res. 2012; 72:3709-3714.

14. Imbalzano AN, Kwon $\mathrm{H}$, Green MR, Kingston RE. Facilitated binding of TATA-binding protein to nucleosomal DNA. Nature. 1994; 370:481-485. 
15. Kwon H, Imbalzano AN, Khavari PA, Kingston RE, Green MR. Nucleosome disruption and enhancement of activator binding by a human SW1/SNF complex. Nature. 1994; 370:477-481.

16. Wang W, Xue Y, Zhou S, Kuo A, Cairns BR, Crabtree GR. Diversity and specialization of mammalian SWI/SNF complexes. Genes Dev. 1996; 10:2117-2130.

17. Versteege I, Sevenet N, Lange J, Rousseau-Merck MF, Ambros P, Handgretinger R, Aurias A, Delattre O. Truncating mutations of hSNF5/INI1 in aggressive paediatric cancer. Nature. 1998; 394:203-206.

18. Wang X, Sansam CG, Thom CS, Metzger D, Evans JA, Nguyen PT, Roberts CW. Oncogenesis caused by loss of the SNF5 tumor suppressor is dependent on activity of BRG1, the ATPase of the SWI/SNF chromatin remodeling complex. Cancer Res. 2009; 69:8094-8101.

19. Kadoch C, Hargreaves DC, Hodges C, Elias L, Ho L, Ranish J, Crabtree GR. Proteomic and bioinformatic analysis of mammalian SWI/SNF complexes identifies extensive roles in human malignancy. Nat Genet. 2013; 45:592-601.

20. Shain AH, Pollack JR. The spectrum of SWI/SNF mutations, ubiquitous in human cancers. PLoS One. 2013; 8:e55119.

21. Cohet N, Stewart KM, Mudhasani R, Asirvatham AJ, Mallappa C, Imbalzano KM, Weaver VM, Imbalzano AN, Nickerson JA. SWI/SNF chromatin remodeling enzyme ATPases promote cell proliferation in normal mammary epithelial cells. J Cell Physiol. 2010; 223:667-678.

22. Wu Q, Madany P, Akech J, Dobson JR, Douthwright S, Browne G, Colby JL, Winter GE, Bradner JE, Pratap J, Sluder G, Bhargava R, Chiosea SI, et al. The SWI/SNF ATPases Are Required for Triple Negative Breast Cancer Cell Proliferation. J Cell Physiol. 2015; 230:2683-2694.

23. Bai J, Mei P, Zhang C, Chen F, Li C, Pan Z, Liu H, Zheng J. BRG1 is a prognostic marker and potential therapeutic target in human breast cancer. PLoS One. 2013; 8:e59772.

24. Jones RG, Thompson CB. Tumor suppressors and cell metabolism: a recipe for cancer growth. Genes Dev. 2009; 23:537-548.

25. Cantor JR, Sabatini DM. Cancer cell metabolism: one hallmark, many faces. Cancer Discov. 2012; 2:881-898.

26. Dang CV. Links between metabolism and cancer. Genes Dev. 2012; 26:877-890.

27. Imbalzano KM, Cohet $\mathrm{N}$, Wu Q, Underwood JM, Imbalzano AN, Nickerson JA. Nuclear shape changes are induced by knockdown of the SWI/SNF ATPase BRG1 and are independent of cytoskeletal connections. PLoS One. 2013; 8:e55628.

28. Dutta P, Tanti GK, Sharma S, Goswami SK, Komath SS, Mayo MW, Hockensmith JW, Muthuswami R. Global epigenetic changes induced by SWI2/SNF2 inhibitors characterize neomycin-resistant mammalian cells. PLoS One. 2012; 7:e49822.
29. Muthuswami R, Mesner LD, Wang D, Hill DA, Imbalzano AN, Hockensmith JW. Phosphoaminoglycosides inhibit SWI2/SNF2 family DNA-dependent molecular motor domains. Biochemistry. 2000; 39:4358-4365.

30. Wu Q, Sharma S, Cui H, LeBlanc SE, Zhang H, Muthuswami R, Nickerson JA, Imbalzano AN. Targeting the chromatin remodeling enzyme BRG1 increases the efficacy of chemotherapy drugs in breast cancer cells. Oncotarget. 2016; 7:27158-27175. doi: 10.18632/oncotarget.8384.

31. Abramson HN. The lipogenesis pathway as a cancer target. J Med Chem. 2011; 54:5615-5638.

32. Elshourbagy NA, Near JC, Kmetz PJ, Wells TN, Groot PH, Saxty BA, Hughes SA, Franklin M, Gloger IS. Cloning and expression of a human ATP-citrate lyase cDNA. Eur J Biochem. 1992; 204:491-499.

33. Ghosh B, Barbosa E, Singh I. Molecular cloning and sequencing of human palmitoyl-CoA ligase and its tissue specific expression. Mol Cell Biochem. 1995; 151:77-81.

34. Hall AM, Smith AJ, Bernlohr DA. Characterization of the Acyl-CoA synthetase activity of purified murine fatty acid transport protein 1. J Biol Chem. 2003; 278:43008-43013.

35. Krammer J, Digel M, Ehehalt F, Stremmel W, Fullekrug J, Ehehalt R. Overexpression of CD36 and acyl-CoA synthetases FATP2, FATP4 and ACSL1 increases fatty acid uptake in human hepatoma cells. Int J Med Sci. 2011; 8:599-614.

36. Han GS, Carman GM. Characterization of the human LPIN1-encoded phosphatidate phosphatase isoforms. J Biol Chem. 2010; 285:14628-14638.

37. Rosenbloom KR, Dreszer TR, Pheasant M, Barber GP, Meyer LR, Pohl A, Raney BJ, Wang T, Hinrichs AS, Zweig AS, Fujita PA, Learned K, Rhead B, et al. ENCODE whole-genome data in the UCSC Genome Browser. Nucleic Acids Res. 2010; 38:D620-625.

38. de la Serna IL, Ohkawa Y, Berkes CA, Bergstrom DA, Dacwag CS, Tapscott SJ, Imbalzano AN. MyoD targets chromatin remodeling complexes to the myogenin locus prior to forming a stable DNA-bound complex. Mol Cell Biol. 2005; 25:3997-4009.

39. Fryer CJ, Archer TK. Chromatin remodelling by the glucocorticoid receptor requires the BRG1 complex. Nature. 1998; 393:88-91.

40. Salma N, Xiao H, Mueller E, Imbalzano AN. Temporal recruitment of transcription factors and SWI/SNF chromatin-remodeling enzymes during adipogenic induction of the peroxisome proliferator-activated receptor gamma nuclear hormone receptor. Mol Cell Biol. 2004; 24:4651-4663.

41. Halvorson DL, McCune SA. Inhibition of fatty acid synthesis in isolated adipocytes by 5-(tetradecyloxy)-2furoic acid. Lipids. 1984; 19:851-856.

42. Kuhajda FP, Pizer ES, Li JN, Mani NS, Frehywot GL, Townsend CA. Synthesis and antitumor activity of an inhibitor of fatty acid synthase. Proc Natl Acad Sci U S A. 2000; 97:3450-3454. 
43. Brusselmans K, De Schrijver E, Verhoeven G, Swinnen JV. RNA interference-mediated silencing of the acetyl-CoAcarboxylase-alpha gene induces growth inhibition and apoptosis of prostate cancer cells. Cancer Res. 2005; 65:6719-6725.

44. Currie E, Schulze A, Zechner R, Walther TC, Farese RV Jr. Cellular fatty acid metabolism and cancer. Cell Metab. 2013; 18:153-161.

45. Kuhajda FP. Fatty acid synthase and cancer: new application of an old pathway. Cancer Res. 2006; 66:5977-5980.

46. Jayakumar A, Tai MH, Huang WY, al-Feel W, Hsu M, AbuElheiga L, Chirala SS, Wakil SJ. Human fatty acid synthase: properties and molecular cloning. Proc Natl Acad Sci USA. 1995; 92:8695-8699.

47. Rudolph MC, McManaman JL, Hunter L, Phang T, Neville MC. Functional development of the mammary gland: use of expression profiling and trajectory clustering to reveal changes in gene expression during pregnancy, lactation, and involution. J Mammary Gland Biol Neoplasia. 2003; 8:287-307.

48. Sturmey RG, Reis A, Leese HJ, McEvoy TG. Role of fatty acids in energy provision during oocyte maturation and early embryo development. Reprod Domest Anim. 2009; 44 Suppl 3:50-58.

49. Kersten S. Mechanisms of nutritional and hormonal regulation of lipogenesis. EMBO Rep. 2001; 2:282-286.

50. Flavin R, Peluso S, Nguyen PL, Loda M. Fatty acid synthase as a potential therapeutic target in cancer. Future Oncol. 2010; 6:551-562.

51. Gabrielson EW, Pinn ML, Testa JR, Kuhajda FP. Increased fatty acid synthase is a therapeutic target in mesothelioma. Clin Cancer Res. 2001; 7:153-157.

52. Khwairakpam AD, Shyamananda MS, Sailo BL, Rathnakaram SR, Padmavathi G, Kotoky J, Kunnumakkara AB. ATP citrate lyase (ACLY): a promising target for cancer prevention and treatment. Curr Drug Targets. 2015; 16:156-163.

53. Corominas-Faja B, Cuyas E, Gumuzio J, Bosch-Barrera J, Leis O, Martin AG, Menendez JA. Chemical inhibition of acetyl-CoA carboxylase suppresses self-renewal growth of cancer stem cells. Oncotarget. 2014; 5:8306-8316. doi: 10.18632/oncotarget.2059.

54. Wang C, Ma J, Zhang N, Yang Q, Jin Y, Wang Y. The acetylCoA carboxylase enzyme: a target for cancer therapy? Expert Rev Anticancer Ther. 2015; 15:667-676.

55. Imbalzano AN, Imbalzano KM, Nickerson JA. BRG1, a SWI/SNF chromatin remodeling enzyme ATPase, is required for maintenance of nuclear shape and integrity. Communicative \& integrative biology. 2013; 6:e25153.

56. Medina PP, Romero OA, Kohno T, Montuenga LM, Pio R, Yokota J, Sanchez-Cespedes M. Frequent BRG1/ SMARCA4-inactivating mutations in human lung cancer cell lines. Hum Mutat. 2008; 29:617-622.
57. Wilson BG, Roberts CW. SWI/SNF nucleosome remodellers and cancer. Nat Rev Cancer. 2011; 11:481-492.

58. Hoffman GR, Rahal R, Buxton F, Xiang K, McAllister G, Frias E, Bagdasarian L, Huber J, Lindeman A, Chen D, Romero R, Ramadan N, Phadke T, et al. Functional epigenetics approach identifies BRM/SMARCA2 as a critical synthetic lethal target in BRG1-deficient cancers. Proc Natl Acad Sci U S A. 2014; 111:3128-3133.

59. Oike T, Ogiwara H, Tominaga Y, Ito K, Ando O, Tsuta K, Mizukami T, Shimada Y, Isomura H, Komachi M, Furuta K, Watanabe S, Nakano T, et al. A synthetic lethality-based strategy to treat cancers harboring a genetic deficiency in the chromatin remodeling factor BRG1. Cancer Res. 2013; 73:5508-5518.

60. Fillmore CM, Xu C, Desai PT, Berry JM, Rowbotham SP, Lin YJ, Zhang H, Marquez VE, Hammerman PS, Wong KK, Kim CF. EZH2 inhibition sensitizes BRG1 and EGFR mutant lung tumours to TopoII inhibitors. Nature. 2015; 520:239-242.

61. Jubierre L, Soriano A, Planells-Ferrer L, Paris-Coderch L, Tenbaum SP, Romero OA, Moubarak RS, AlmazanMoga A, Molist C, Roma J, Navarro S, Noguera R, Sanchez-Cespedes M, et al. BRG1/SMARCA4 is essential for neuroblastoma cell viability through modulation of cell death and survival pathways. Oncogene. 2016.

62. Liu X, Tian X, Wang F, Ma Y, Kornmann M, Yang Y. BRG1 promotes chemoresistance of pancreatic cancer cells through crosstalking with Akt signalling. Eur J Cancer. 2014; 50:2251-2262.

63. Saladi SV, Keenen B, Marathe HG, Qi H, Chin KV, de la Serna IL. Modulation of extracellular matrix/adhesion molecule expression by BRG1 is associated with increased melanoma invasiveness. Mol Cancer. 2010; 9:280.

64. Sentani K, Oue N, Kondo H, Kuraoka K, Motoshita J, Ito R, Yokozaki H, Yasui W. Increased expression but not genetic alteration of BRG1, a component of the SWI/SNF complex, is associated with the advanced stage of human gastric carcinomas. Pathobiology. 2001; 69:315-320.

65. Shi J, Whyte WA, Zepeda-Mendoza CJ, Milazzo JP, Shen C, Roe JS, Minder JL, Mercan F, Wang E, EckersleyMaslin MA, Campbell AE, Kawaoka S, Shareef S, et al. Role of SWI/SNF in acute leukemia maintenance and enhancer-mediated Myc regulation. Genes Dev. 2013; 27:2648-2662.

66. Sun A, Tawfik O, Gayed B, Thrasher JB, Hoestje S, Li C, Li B. Aberrant expression of SWI/SNF catalytic subunits BRG1/BRM is associated with tumor development and increased invasiveness in prostate cancers. Prostate. 2007; 67:203-213.

67. Watanabe T, Semba S, Yokozaki H. Regulation of PTEN expression by the SWI/SNF chromatin-remodelling protein BRG1 in human colorectal carcinoma cells. Br J Cancer. 2011; 104:146-154.

68. Debnath J, Muthuswamy SK, Brugge JS. Morphogenesis and oncogenesis of MCF-10A mammary epithelial acini 
grown in three-dimensional basement membrane cultures. Methods. 2003; 30:256-268.

69. Guise TA, Yin JJ, Taylor SD, Kumagai Y, Dallas M, Boyce BF, Yoneda T, Mundy GR. Evidence for a causal role of parathyroid hormone-related protein in the pathogenesis of human breast cancer-mediated osteolysis. The Journal of clinical investigation. 1996; 98:1544-1549.
70. de La Serna IL, Carlson KA, Hill DA, Guidi CJ, Stephenson RO, Sif S, Kingston RE, Imbalzano AN. Mammalian SWI-SNF complexes contribute to activation of the hsp70 gene. Mol Cell Biol. 2000; 20:2839-2851.

71. Bligh EG, Dyer WJ. A rapid method of total lipid extraction and purification. Can J Biochem Physiol. 1959; 37:911-917. 